\title{
Proteomic analysis of plasma profiles in children with recurrent bone fractures
}

\author{
Agnieszka Rusińska ${ }^{凶}$, Maria Świątkowska², Wiktor Koziołkiewicz², Szymon Skurzyński², \\ Joanna Golec ${ }^{1}$ and Danuta Chlebna-Sokół'
}

1Department of Paediatric Propedeutics and Bone Metabolic Diseases, Medical University of Lodz, Lodz, Poland; ${ }^{2}$ Department of Molecular and Medical Biophysics, Medical University of Lodz, Lodz, Poland

\begin{abstract}
The aim of the study is proteomic analysis of the plasma profile in children with recurrent bone fractures. The study involved 16 children: 6 patients with recurrent low-energy fractures and normal bone mass and 10 with osteogenesis imperfecta. In the analysis of the protein profile, the two-dimensional protein electrophoresis was used (Ettan DALT II, Amersham Bioscience). The images of protein gels were compared with controls. The protein spots with changed expression were cut from the gel and the amino acid sequence was analyzed with the mass spectrometry method (Q-Tof Premier ${ }^{\mathrm{TM}}$ API MASS SPECTROMETR, Waters) for protein identification. The most prevalent protein with changed expression, with respect to controls, was haptoglobin observed in 6 patients with a severe form of osteogenesis imperfecta. Increased haptoglobin concentration in these patients was confirmed by the ELISA method. Peptides corresponding to alpha- 1 acid glycoprotein and serum amyloid $P$ component, apolipoprotein A-I, and transthyretin were detected in one, two and three children, respectively. Conclusions: 1) The results show increased haptoglobin which may be suggestive of an inflammatory component taking part in the course of osteogenesis imperfecta. 2) Further studies to explain the possible relationship of this protein with increased bone fragility are necessary.
\end{abstract}

Keywords: bone fractures, proteomic plasma profile

Received: 10 May, 2011; revised: 24 November, 2011; accepted: 29 November, 2011; available on-line: 1 December, 2011

\section{INTRODUCTION}

Recurrent bone fractures are a serious paediatric problem (Khosla et al., 2003). The results of own studies conducted to date as well as observations of other authors show that most frequently they are not only the effect of increased incidence of injuries in the developmental age, but also a symptom of bone mineralization or structure disturbances (Manias et al., 2006). Many fractures in children may be a consequence of decreased bone mineral density or abnormalities in calcium and phosphate balance and bone metabolism. In some cases, however, bone mass corresponding to skeletal mineralization is normal, and still repeated fractures occur in these patients. In such circumstances abnormal bone tissue composition or structure is suspected and diagnostics as well as treatment of these disturbances are particularly difficult (Clark et al., 2006). The main component of the organic bone part are proteins - 90\% collagen, and the other $10 \%$ - non-collagen proteins. Direct evaluation of proteins in the bone tissue is difficult due to the necessity of invasive sampling of this tissue for histomorphometric analysis (Pernow et al., 2009). Bone is however a metabolically active tissue and in the process of bone remodelling both the propeptides subsequently forming its structure and the bone protein degradation products enter the blood. To date, only quantitative assessment of some proteins - commonly accepted bone formation and bone resorption markers - in the serum was performed (Cundy et al., 2007). In the recent years, owing to the dynamic development of molecular biology, a possibility of qualitative assessment of proteins with proteomic methods emerged and first attempts have already been made to use this technique for studies on the pathogenesis of some motor system conditions (MayerKuckuk et al., 2006; de Ceunick, 2008; Zheng et al., 2009; Zhang et al., 2010). However no papers concerning qualitative assessment of proteins circulating in the blood in patients with recurrent fractures have been published to date, although such publications might contribute to an indirect, deepened analysis of the composition and structure of the organic component of the bone tissue. Only few authors dealt with protein expression in in vivo studies of osteogenesis imperfecta in a mouse model, however no such analyses were done in humans (Forlino et al., 2007). Therefore research was undertaken whose purpose is to try to explain the aetiopathogenesis of repeated bone fractures based on a complex qualitative plasma protein profile analysis in children with recurrent fractures of unknown aetiology (RF) and in patients with osteogenesis imperfecta (OI).

\section{MATERIALS AND METHODS}

The study involved 16 children, including 6 patients with recurrent low-energy bone fractures (RF) not related to a decrease in bone mass (aged 12-16 years, number of fractures ranging from 4 to 7) and $10 \mathrm{pa}-$ tients with diagnosed osteogenesis imperfecta (OI type I in 4 children - OIt1, type III in 6 children - OIt3;

e-mail: agnieszka.rusinska@wp.pl

Preliminary report: International Osteoporosis Foundation World Congress of Osteoporosis, Florence, Italy, 2010 (Osteoporosis International 2010, 21, Suppl. 1:S299). Third Central European Congress on Osteoporosis and Osteoarthritis, Kraków, Poland, 2009 (Ortopedia, Traumatologia, Rehabilitacja 2009, Suppl. 2: 175-176)

Abbreviations: RF, recurrent fractures; Ol, osteogenesis imperfecta; Olt1, osteogenesis imperfecta type I; Olt3, osteogenesis imperfecta type III. 
age: 4 months -15 years). The tests were done at least 2 months after the occurrence of the last fracture. Active inflammatory conditions were excluded in all children: no clinical symptoms of inflammation were present, Creactive protein levels assessed by a turbidimetric method and quantitative serum electrophoresis were normal. The study enrolled patients in whom chronic diseases promoting secondary skeletal mineralisation disturbances were excluded (diseases requiring long-term systemic glucocorticoid therapy, diseases of endocrine glands, autoimmune diseases, systemic diseases, intestinal malabsorption syndromes, among others). The results were compared with proteomic plasma profile of 5 healthy children without previous fractures (controls - C). Clinical, densitometric and biochemical characteristics of the studied patients are shown in Table 1 and Table 2. Bone turnover markers were assessed by commercially available ELISA tests, liver metabolite of vitamin D was determined by a radiocompetitive method and parathormone by chemiluminescence.

The study obtained approval of the Bioethics Committee of the Medical University of Lódź (No.
RNN/24/07/KE). Parents provided written consent for participation of their children in the study.

Fasting blood samples for tests were taken in the morning, to a tube with EDTA, and they were subsequently centrifuged at $1500 \mathrm{rpm}$ for 15 minutes. Plasma samples obtained were stored at the temperature $-70^{\circ} \mathrm{C}$ to the time of analysis.

Two-dimensional polyacrylamide gel electrophoresis. Proteins were separated by two-dimensional electrophoresis, using ready-made gels with immobilized $\mathrm{pH}$ gradients (Amersham-Biosciences). Albumin and $\operatorname{IgG}$ were removed from samples of human plasma using ProteoPreb Blue Albumin and IgG Depletion Kit (Sigma). For the first dimension, samples containing approximately $70 \mu \mathrm{g}$ of soluble protein in lysis buffer were mixed with IPG Resewelling Solution (8 M urea, 1\% Chaps, 0.4\% DTT, 0.5\% Pharmalyte) to obtain a final volume of $450 \mu \mathrm{l}$. They were then loaded onto $24 \mathrm{~cm}$ immobilized $\mathrm{pH}$ linear gradient strip gels (IPG, pH 4-7). IEF strips were allowed to rehydrate for $5 \mathrm{~h}$, and isoelectric focusing was performed according to a protocol by gradual increase of

Table 1. Clinical characteristic of studied children

\begin{tabular}{|c|c|c|c|c|c|}
\hline $\begin{array}{l}\text { Diagnosis } \\
\text { (Patient initials) }\end{array}$ & $\begin{array}{l}\text { Age } \\
\text { (month } \\
\text { or year }^{b} \text { ) }\end{array}$ & Gender & Localisation/number of previous bone fractures & $\begin{array}{l}\text { Time from the } \\
\text { last fracture } \\
\text { (min) }\end{array}$ & $\begin{array}{l}\text { Other skeletal } \\
\text { symptoms }\end{array}$ \\
\hline \multicolumn{6}{|c|}{ Recurrent fractures and normal bone mass (RF) } \\
\hline $\mathrm{RF}(\mathrm{DM})$ & 12 years & $\mathrm{F}$ & forearm, phalanges / 7 & 2 & none \\
\hline $\mathrm{RF}(\mathrm{KP})$ & 13 years & M & forearm, phalanges / 4 & 36 & none \\
\hline $\mathrm{RF}(\mathrm{T} 七)$ & 14 years & M & forearm, phalanges, crural, metatarsal / 5 & 5 & none \\
\hline $\mathrm{RF}(\mathrm{CD})$ & 14 years & M & humeral, clavicle, scapula, vertebrae, femoral / 6 & 3 & back and legs pain \\
\hline $\mathrm{RF}(\mathrm{BM})$ & 15 years & M & forearm, metatarsal, phalanges / 6 & 8 & back pain \\
\hline $\mathrm{RF}(\mathrm{SP})$ & 16 years & M & phalanges / 7 & 36 & back pain \\
\hline \multicolumn{6}{|c|}{ Osteogenesis imperfecta type I (Olt1) } \\
\hline Olt1(ŚN) & 3 years & M & forearm, phalanges, femoral, iliac / 5 & 8 & arms and legs pain \\
\hline Olt1(GW) & 7 years & M & forearm, humeral / 6 & 12 & legs pain \\
\hline Olt1(KK) & 8 years & $M$ & forearm, vertebrae, ribs / 7 & 12 & legs pain \\
\hline Olt1(TA) & 15 years & M & forearm, humeral, clavicle, crural / 8 & 8 & legs pain \\
\hline \multicolumn{6}{|c|}{ Osteogenesis imperfecta type III (Olt3) } \\
\hline Olt3(MW) & 4 months & $\mathrm{F}$ & $\begin{array}{l}\text { forearm, humeral, femoral, crural, ribs / many fractures } \\
\text { in fetal period }\end{array}$ & 4 & $\begin{array}{l}\text { bone deformation } \\
\text { and pain }\end{array}$ \\
\hline Olt3(KZ) & 9 months & $\mathrm{F}$ & $\begin{array}{l}\text { forearm, humeral, femoral, ribs } / 7+\text { many fractures in } \\
\text { fetal period }\end{array}$ & 6 & $\begin{array}{l}\text { bone deformation } \\
\text { and pain }\end{array}$ \\
\hline Olt3(OM) & 2 years & $\mathrm{F}$ & $\begin{array}{l}\text { cranial, femoral, crural / } 6+\text { many fractures in fetal } \\
\text { period }\end{array}$ & 6 & $\begin{array}{l}\text { bone deformation } \\
\text { and pain }\end{array}$ \\
\hline Olt3(PP) & 4 years & $\mathrm{F}$ & $\begin{array}{l}\text { forearm, humeral, femoral, crural, ribs, vertebrae / } 40+ \\
\text { many fractures in fetal period }\end{array}$ & 2 & $\begin{array}{l}\text { bone deformation } \\
\text { and pain }\end{array}$ \\
\hline Olt3(MA) & 1 year & M & $\begin{array}{l}\text { forearm, humeral, femoral, crural / 1+ many fractures in } \\
\text { fetal period }\end{array}$ & 11 & $\begin{array}{l}\text { bone deformation } \\
\text { and pain }\end{array}$ \\
\hline Olt3(NB) & 2 years & $M$ & $\begin{array}{l}\text { humeral, clavicle, femoral / } 9+\text { many fractures in fetal } \\
\text { period }\end{array}$ & 3 & $\begin{array}{l}\text { bone deformation } \\
\text { and pain }\end{array}$ \\
\hline \multicolumn{6}{|c|}{ Controls without fractures(C) } \\
\hline $\mathrm{C}(\mathrm{JA})$ & 6 years & $\mathrm{F}$ & none & - & none \\
\hline$C(S A)$ & 1 month & $M$ & none & - & none \\
\hline$C(D P)$ & 2 years & $M$ & none & - & none \\
\hline$C(\mathrm{RJ})$ & 11 years & $M$ & none & - & none \\
\hline C(NM) & 17 years & M & none & - & none \\
\hline
\end{tabular}


Table 2. Densitometric and biochemical characteristic of studied children

\begin{tabular}{|c|c|c|c|c|c|c|c|c|c|c|}
\hline $\begin{array}{l}\text { Diagnosis } \\
\text { (Patient initials) }\end{array}$ & $\begin{array}{l}\text { Total body } \\
\text { BMD } \\
\mathrm{g} / \mathrm{cm}^{2} \\
\text { (Z-score) }\end{array}$ & $\begin{array}{l}\text { Spine L2L4 } \\
\text { BMD } \\
\mathrm{g} / \mathrm{cm}^{2} \\
\text { (Z-score) }\end{array}$ & $\begin{array}{l}25 \mathrm{OHD} \\
\mathrm{ng} / \mathrm{ml}\end{array}$ & $\begin{array}{l}\text { PTH } \\
\mathrm{pg} / \mathrm{ml}\end{array}$ & $\begin{array}{l}\mathrm{OC} \\
\mathrm{ng} / \mathrm{ml}\end{array}$ & $\begin{array}{l}\text { PINP } \\
\mathrm{ng} / \mathrm{ml}\end{array}$ & $\begin{array}{l}\text { CTX } \\
\mathrm{ng} / \mathrm{ml}\end{array}$ & $\begin{array}{l}\text { NTX } \\
\text { mMBCE/mM } \\
\text { creatinine }\end{array}$ & $\begin{array}{l}\text { OPG } \\
\mathrm{pmol} / \mathrm{l}\end{array}$ & $\begin{array}{l}\text { sRANKL } \\
\mathrm{pmol} / \mathrm{l}\end{array}$ \\
\hline \multicolumn{11}{|c|}{ Recurrent fractures and normal bone mass (RF) } \\
\hline $\mathrm{RF}(\mathrm{DM})$ & $0.969(0.0)$ & $\begin{array}{l}0.898 \\
(-0.3)\end{array}$ & 40.3 & 34.0 & 160.4 & 364.3 & 1.8 & 399 & 4.0 & 0.0 \\
\hline $\mathrm{RF}(\mathrm{KP})$ & $\begin{array}{l}0.648 \\
(-0.2)\end{array}$ & $\begin{array}{l}0.853 \\
(-0.4)\end{array}$ & 29.8 & 24.7 & 139.3 & 375.7 & 1.5 & 1080 & 4.5 & 0.0 \\
\hline $\mathrm{RF}(\mathrm{T} Ł)$ & $\begin{array}{l}0.980 \\
(0.5)\end{array}$ & $\begin{array}{l}0.953 \\
(-0.4)\end{array}$ & 46.7 & 18.8 & 99.1 & 244.9 & 1.8 & 1604 & 3.9 & 0.0 \\
\hline $\mathrm{RF}(\mathrm{CD})$ & $\begin{array}{l}1.011 \\
(1.3)\end{array}$ & $\begin{array}{l}0.925 \\
(-0.1)\end{array}$ & 33.1 & 48.4 & 135.9 & 327.1 & 1.8 & 162 & 2.6 & 0.3 \\
\hline $\mathrm{RF}(\mathrm{BM})$ & $\begin{array}{l}0.970 \\
(0.1)\end{array}$ & $\begin{array}{l}0.923 \\
(-0.8)\end{array}$ & 38.0 & 27.0 & 93.8 & 178.1 & 1.2 & 444 & 2.5 & 0.1 \\
\hline $\mathrm{RF}(\mathrm{SP})$ & $\begin{array}{l}1.077 \\
(-0.8)\end{array}$ & $\begin{array}{l}1.116 \\
(-0.7)\end{array}$ & 23.2 & 33.1 & 111.0 & 213.7 & 2.2 & 290 & 2.5 & 0.0 \\
\hline \multicolumn{11}{|c|}{ Osteogenesis imperfecta type I (Olt1) } \\
\hline Olt1(ŚN) & $\begin{array}{l}0,437 \\
(-1,8)\end{array}$ & - & 75.8 & 8.6 & 107.3 & 290.2 & 0.4 & 630 & 2.5 & 0.0 \\
\hline Olt1(GW) & $\begin{array}{l}0.748 \\
(-1.5)\end{array}$ & $\begin{array}{l}0.428 \\
(-3.1)\end{array}$ & 22.6 & 27.5 & 124.8 & 247.4 & 0.5 & 869 & 2.1 & 0.1 \\
\hline Olt1(KK) & $\begin{array}{l}0.835 \\
(-1,5)\end{array}$ & - & 44.8 & 51.4 & 142.9 & 266.8 & 0.8 & 599 & 4.0 & 0.3 \\
\hline Olt1(TA) & $\begin{array}{l}0.704 \\
(-2.8) \\
\end{array}$ & $\begin{array}{l}0.596 \\
(-3.5)\end{array}$ & 9.5 & 21.6 & 113.6 & 384.5 & 1.7 & 509 & 4.5 & 0.0 \\
\hline \multicolumn{11}{|c|}{ Osteogenesis imperfecta type III (Olt3) } \\
\hline Olt3(MW) & $\begin{array}{l}0.327 \\
(-1.4)\end{array}$ & - & 34.3 & 9.8 & 26.5 & 287.2 & 0.3 & 312 & 6.3 & 0.0 \\
\hline Olt3(KZ) & $\begin{array}{l}0.419 \\
(-1.6)\end{array}$ & - & 70.5 & 15.3 & 41.7 & 193.1 & 0.4 & 306 & 5.5 & 0.0 \\
\hline Olt3(OM) & - & - & 41.5 & 19.2 & 126.1 & 259.1 & 0.2 & 330 & 4.5 & 0.0 \\
\hline Olt3(PP) & $\begin{array}{l}0.454 \\
(-3.8)\end{array}$ & - & 73.2 & 29.2 & 43.0 & 73.2 & 0.3 & 390 & 5.5 & 0.0 \\
\hline Olt3(MA) & $\begin{array}{l}0.492 \\
(-1,9)\end{array}$ & - & 42.2 & 25.7 & 36.1 & 184.3 & 0.4 & 1314 & 3.4 & 0.1 \\
\hline Olt3(NB) & $\begin{array}{l}0.726 \\
(3.1)\end{array}$ & - & 40.3 & 30.3 & 38.6 & 147.9 & 0.3 & 396 & 4.8 & 0.0 \\
\hline
\end{tabular}

Abbreviations: BMD, bone mineral density by dual energy X-ray absorptiometry; 25OHD, serum liver metabolite of vitamin $\mathrm{D}$; $\mathrm{PTH}$, serum parathormon, OC, serum osteocalcin; PINP, serum N-terminal propeptide of procollagen type I; CTX, serum C-terminal telopeptide of collagen type I; NTX, urine N-terminal telopeptide of collagen type I; OPG, serum osteoprotegerin; sRANKL, serum soluble receptor activator of nuclear factor kappa B ligand.

voltage $(30 \mathrm{~V}$ for $5 \mathrm{~h}, 500 \mathrm{~V}$ for $1 \mathrm{~h}, 1000 \mathrm{~V}$ for $1 \mathrm{~h}$, followed by $60 \mathrm{kVh}$ at $8000 \mathrm{~V}$ ), using an Ettan IPGphor system (Amersham Bioscience). Before the second dimension of electrophoresis the strips were equilibrated for 15 minutes in a solution containing 65 mmol DTT $6 \mathrm{M}$ urea $30 \%$ glycerol $2 \%$ SDS and 50 mmol Tris/HCL (pH 6.8). Thereafter, they were subjected to a new equilibration solution for 15 minutes where DTT was replaced by $243 \mathrm{mmol}$ iodoacetamide. Second-dimension SDS electrophoresis was performed on $12.5 \%$ polyacrylamide gels using the Ettan Dalt II vertical system (Amersham Bioscience). Protein spots were visualized by silver-staining according to the method compatible with the analysis of protein mass spectrometry (Shevchenko et al., 1996). Silver-stained gels were digitized using a laser Image Scanner (Amersham-Bioscience). The images of protein gels were compared with controls. Computerized 2D gel analysis was performed with the Image Master 2D software package Version 3.0. Protein spots with changed abundance as well as those that appeared only in patients were excised from the gel subjected to in-gel digestion with trypsin.

Mass spectrometry, data base search, and data processing. Proteins in each gel slice were subjected to reduction with $10 \mathrm{mM}$ dithiothreitol, alkylation with 50 $\mathrm{mM}$ iodoacetamide, and tryptic digestion with sequencing grade modified trypsin $\left(10 \mu \mathrm{g} / \mathrm{mL}\right.$; Promega) at $37^{\circ}$ $\mathrm{C}$ for $14 \mathrm{~h}$. After in-gel digestion, the product peptides were extracted stepwise with two portions of $35 \mu \mathrm{L}$ of $0.1 \%$ trifluoroacetic acid in $2 \%$ acetonitrile and loaded on a RP-C18 nanoAcquity UPLC pre-column (Waters). Peptides were eluted to a C18 nanoAcquity UPLC column $(75 \mu \mathrm{m} \times 25 \mathrm{~cm}$; Waters $)$ by acetonitrile gradient in the presence of formic acid and then directly applied into an electrospray spectrometer (QUADRUPOLE TIMEOF-FLIGHT TANDEM Q-Tof Premier ${ }^{\mathrm{TM}}$ API MASS SPECTROMETR, Waters). Linear acetonitrile gradient and $0.1 \%$ formic acid were used for peptide elution. In spectra acquisition the reflectron status was on $(\mathrm{kV}: 2.04$; 
Table 3. Detailed criteria of protein identification by peptide mass fingerprints and MS/MS sequencing

\begin{tabular}{ll}
\hline Attribute & Value \\
\hline Search Engine Type & PLGS \\
Databanks & $\begin{array}{l}\text { Ident_Swiss_Human } \\
\text { Ident_Trembl_Human }\end{array}$ \\
Species & Human \\
Molecular Weight Range & 0 to 200000 Da \\
pl Range & 0 to 14 \\
Maximum Hits to Return & 50 \\
Primary Digest Reagent & Trypsin \\
Secondary Digest Reagent & None \\
Monoisotopic or Average & Monoisotopic \\
Mass Values & MH+ \\
Peptide Charge & $2+$ \\
\hline
\end{tabular}

$\mu \mathrm{A}: 58$ ), the ionization source was electrospray and the following settings were used: capillary $(\mathrm{kV}) 3.0$; source temperature $\left({ }^{\circ} \mathrm{C}\right)$ 80; desolvation temperature $\left({ }^{\circ} \mathrm{C}\right) 180$. Proteins were identified by peptide mass fingerprinting, adding confidence to the protein identification. Detailed criteria of protein identification both by peptide mass fingerprints and MS/MS sequencing were summarized in Table 3. The Swiss-Prot protein database was searched with the PLGS 2.2.5 program. The list of top candidates for each sample was verified by inspection of the quality of sequencing data. We examined their automatic ordering manually in terms of their reliability scores and MS spectrum profiles to pick up only highly reliable peptide data (sorted data). Parameters used for database searching were the following: precursor-ion mass tolerance $15 \mathrm{ppm}$, fragment mass tolerance $-0.2 \mathrm{Da}$, number of missed cleavages - 1 , variable modifications - carbamidomethyl $\mathrm{C}$, fixed modifications — oxidation M.
Quantitative haptoglobin evaluation. Serum concentration of haptoglobin in children with elevated abundance of this protein in proteomic analysis (OIt3) and in controls was determined by the ELISA method (enzyme-linked immunosorbent assay) with the Haptoglobin Human ELISA kit (ABCAM, UK). The sensitivity threshold of this method was $<20 \mathrm{ng} / \mathrm{ml}$, intra-assay coefficient of variability (CV) was $5.1 \%$, inter-assay was $8.0 \%$. Reference values for healty subjects were $0.3-2$ $\mathrm{g} / \mathrm{l}$. The statistical analysis was made by means of the Statistica 6.0 software. The results were accepted as statistically significant at $p<0.05$.

\section{RESULTS}

In the studied group of patients proteins were detected whose expression was changed with respect to controls as well as proteins that appeared only in the individuals affected by recurrent fractures. Among the spots tested to date, peptides corresponding to the alpha and beta chains of human haptoglobin protein occurred most frequently - in all six children (100\%) with type III osteogenesis imperfecta (Table 4, Fig. 1). On the contrary, the spots corresponding to the above mentioned proteins were found neither in children with recurrent fractures of unknown aetiology and with type I osteogenesis imperfecta nor in the control group. Additionally, in three patients, including two with multiple recurrent fractures and one with type III osteogenesis imperfecta, peptides corresponding to the human transthyretin protein were found (Table 4, Fig. 2). Another repeatedly found protein was human apolipoprotein A-I in two children (Table 4), including one with recurrent fractures (Fig. 2) and one with type III osteogenesis imperfecta. Additionally, in one of the patients with type III osteogenesis imperfecta peptides corresponding to human serum amyloid P-component were found.

Table 4. Proteins identified in plasma from children with recurrent fractures of unknown aetiology and with osteogenesis imperfecta type III

\begin{tabular}{|c|c|c|c|c|c|c|c|c|}
\hline $\begin{array}{l}\text { Diagnosis } \\
\text { (patient } \\
\text { initials) }\end{array}$ & $\begin{array}{l}\text { Gel } \\
\text { slice } \\
\text { No }\end{array}$ & Protein name & $\begin{array}{l}\text { Protein } \\
\text { accesion } \\
\text { No }\end{array}$ & $\begin{array}{l}\text { Protein } \\
\text { mass } \\
(\mathrm{kDa})\end{array}$ & $\begin{array}{l}\text { Peptides } \\
\text { matches }\end{array}$ & $\begin{array}{l}\text { Probability } \\
\text { (\%) }\end{array}$ & $\begin{array}{l}\text { Sequence } \\
\text { coverage } \\
(\%)\end{array}$ & Peptide sequences \\
\hline \multicolumn{9}{|c|}{ Reccurent fractures and normal bone mass (RF) } \\
\hline \multirow[t]{3}{*}{ RF (DM) } & 1 & $\begin{array}{l}\text { human apolipoprotein } \\
\text { A I }\end{array}$ & P02647 & 30,778 & 13 & 100 & 50.2 & $\begin{array}{l}\text { DLATVYVDVLK } \\
\text { DYVSQFEGSALGK } \\
\text { LLDNWDSVTSTFSK } \\
\text { ETEGLR } \\
\text { VQPYLDDFQK } \\
\text { WQEEMELYR } \\
\text { AELQEGAR } \\
\text { LSPLGEEMR } \\
\text { THLAPYSDELR } \\
\text { ATEHLSTLSEK } \\
\text { AKPALEDLR } \\
\text { QGLLPVLESKK } \\
\text { VSFLSALEEYTK }\end{array}$ \\
\hline & 2 & human transthyretin & P02766 & 15,887 & 3 & 100 & 27.9 & $\begin{array}{l}\text { AADDTWEPFASGK } \\
\text { VEIDTK } \\
\text { YTIAALLSPYSYSTTAVVTNPK }\end{array}$ \\
\hline & 3 & human transthyretin & P02766 & 15,887 & 6 & 100 & 52.4 & $\begin{array}{l}\text { GSPAINVAVHVFR } \\
\text { KAADDTWEPFASGK } \\
\text { AADDTWEPFASGK } \\
\text { TSESGELHGLTTEEEFVEGIYK } \\
\text { VEIDTK } \\
\text { YTIAALLSPYSYSTTAVVTNPK }\end{array}$ \\
\hline RF (BM) & 4 & human transthyretin & P02766 & 15,887 & 2 & 100 & 18.8 & $\begin{array}{l}\text { KAADDTWEPFASGK } \\
\text { AADDTWEPFASGK }\end{array}$ \\
\hline
\end{tabular}




\begin{tabular}{|c|c|c|c|c|c|c|c|c|}
\hline \multicolumn{9}{|c|}{ Osteogenesis imperfecta type III (Olt3) } \\
\hline \multirow[t]{5}{*}{$\begin{array}{l}\text { Olt3 } \\
\text { (MW) }\end{array}$} & 5 & $\begin{array}{l}\text { human apolipoprotein } \\
\text { A I }\end{array}$ & P02647 & 30,778 & 19 & 100 & 67.4 & $\begin{array}{l}\text { VKDLATVYVDVLK } \\
\text { DLATVYVDVLK } \\
\text { DYVSQFEGSALGK } \\
\text { LLDNWDSVTSTFSK } \\
\text { EQLGPVTQEFWDNLEK } \\
\text { DLEEVK } \\
\text { VQPYLDDFQK } \\
\text { WQEEMELYR } \\
\text { QKVEPLR } \\
\text { AELQEGAR } \\
\text { LHELQEK } \\
\text { LSPLGEEMR } \\
\text { AHVDALR } \\
\text { THLAPYSDELR } \\
\text { LAEYHAK } \\
\text { ATEHLSTLSEK } \\
\text { AKPALEDLR } \\
\text { QGLLPVLESFK } \\
\text { VSFLSALEEYTK }\end{array}$ \\
\hline & \multirow[t]{2}{*}{6} & $\begin{array}{l}\text { human serum amyloid } \\
\text { P-component }\end{array}$ & P02743 & 25,387 & 8 & 100 & 34.5 & $\begin{array}{l}\text { VFVFPR } \\
\text { AYSDLSR } \\
\text { AYSLFSYNTQGR } \\
\text { DNELLVYK } \\
\text { VGEYSLYIGR } \\
\text { QGYFVEAQPK } \\
\text { IVLGQEQDSYGGK } \\
\text { GYVIIKPLVWV }\end{array}$ \\
\hline & & $\begin{array}{l}\text { human apolipoprotein } \\
\text { A I }\end{array}$ & P02647 & 30,778 & 8 & 100 & 32.6 & $\begin{array}{l}\text { EQLGPVTQEFWDNLEK } \\
\text { DYVSQFEGSALGK } \\
\text { VQPYLDDFQK } \\
\text { THLAPYSDELR } \\
\text { LLDNWDSTSTFSK } \\
\text { VSFLSALEEYTK } \\
\text { WQEEMELYR } \\
\text { ATEHLSTLSEK }\end{array}$ \\
\hline & \multirow[t]{2}{*}{7} & $\begin{array}{l}\text { human haptoglobin } \\
\text { alpha beta chains }\end{array}$ & P00738 & 45,205 & 2 & 100 & 3.7 & $\begin{array}{l}\text { LRTEGDGVYTLNNEK } \\
\text { TEGDGVYTLNNEK }\end{array}$ \\
\hline & & human transthyretin & P02766 & 15,887 & 2 & 100 & 18.8 & $\begin{array}{l}\text { KAADDTWEPFASGK } \\
\text { AADDTWEPFASGK }\end{array}$ \\
\hline \multirow[t]{3}{*}{ Olt3 (KZ) } & 8 & $\begin{array}{l}\text { human haptoglobin } \\
\text { alpha beta chains }\end{array}$ & P00738 & 45,205 & 4 & 100 & 8.4 & $\begin{array}{l}\text { TEGDGVYTLNDK } \\
\text { TEGDGVYTLNDKK } \\
\text { TEGDGVYTLNNEK } \\
\text { NPANPVQR }\end{array}$ \\
\hline & 9 & $\begin{array}{l}\text { human haptoglobin } \\
\text { alpha beta chains }\end{array}$ & P00738 & 45,205 & 6 & 100 & 9.4 & $\begin{array}{l}\text { LRTEGDGVYTLNDK } \\
\text { TEGDGVYTLNDK } \\
\text { TEGDGVYTLNDKK } \\
\text { LRTEGDGVYTLNNEK } \\
\text { TEGDGVYLNNEK } \\
\text { NPANPVQR }\end{array}$ \\
\hline & 10 & $\begin{array}{l}\text { human haptoglobin } \\
\text { alpha beta chains }\end{array}$ & P00738 & 45,205 & 4 & 100 & 8.4 & $\begin{array}{l}\text { TEGDGVYTLNDK } \\
\text { TEGDGVYTLNDKK } \\
\text { TEGDGVYTLNNEK } \\
\text { NPANPVQR }\end{array}$ \\
\hline Olt3 (OM) & 11 & $\begin{array}{l}\text { human haptoglobin } \\
\text { alpha beta chains }\end{array}$ & P00738 & 45,205 & 2 & 100 & 6.2 & $\begin{array}{l}\text { TEGDGVYTLNDK } \\
\text { TEGDGVYTLNNEK }\end{array}$ \\
\hline Olt3 (PP) & 12 & $\begin{array}{l}\text { human haptoglobin } \\
\text { alpha beta chains }\end{array}$ & P00738 & 45,205 & 6 & 100 & 8.6 & $\begin{array}{l}\text { LRTEGDGVYTLNDK } \\
\text { TEGDGVYTLNDK } \\
\text { TEGDGVYTLNDKK } \\
\text { KQWINK } \\
\text { LRTEGDGVYTLNNEK } \\
\text { TEGDGVYTLNNEK }\end{array}$ \\
\hline \multirow[t]{2}{*}{ Olt3 (MA) } & 13 & $\begin{array}{l}\text { human haptoglobin } \\
\text { alpha beta chains }\end{array}$ & P00738 & 45,205 & 3 & 98.5 & 6.7 & $\begin{array}{l}\text { TEGDGVYTLNDK } \\
\text { LRTEGDGVYTLNNEK } \\
\text { TEGDGVYTLNNEK }\end{array}$ \\
\hline & 14 & $\begin{array}{l}\text { human haptoglobin } \\
\text { alpha beta chains }\end{array}$ & P00738 & 45,205 & 2 & 100 & 3.7 & $\begin{array}{l}\text { LRTEGDGVYTLNNEK } \\
\text { TEGDGVYTLNNEK }\end{array}$ \\
\hline Olt3 (NB) & 15 & $\begin{array}{l}\text { human haptoglobin } \\
\text { alpha beta chain }\end{array}$ & P00738 & 45,207 & 7 & 100 & 7.9 & $\begin{array}{l}\text { STMQELNSR } \\
\text { LASYLDK } \\
\text { MTLDDFR } \\
\text { QGVDADINGLR } \\
\text { EVTQLR } \\
\text { TEGDGVYTLNDKK } \\
\text { KQWINK }\end{array}$ \\
\hline
\end{tabular}



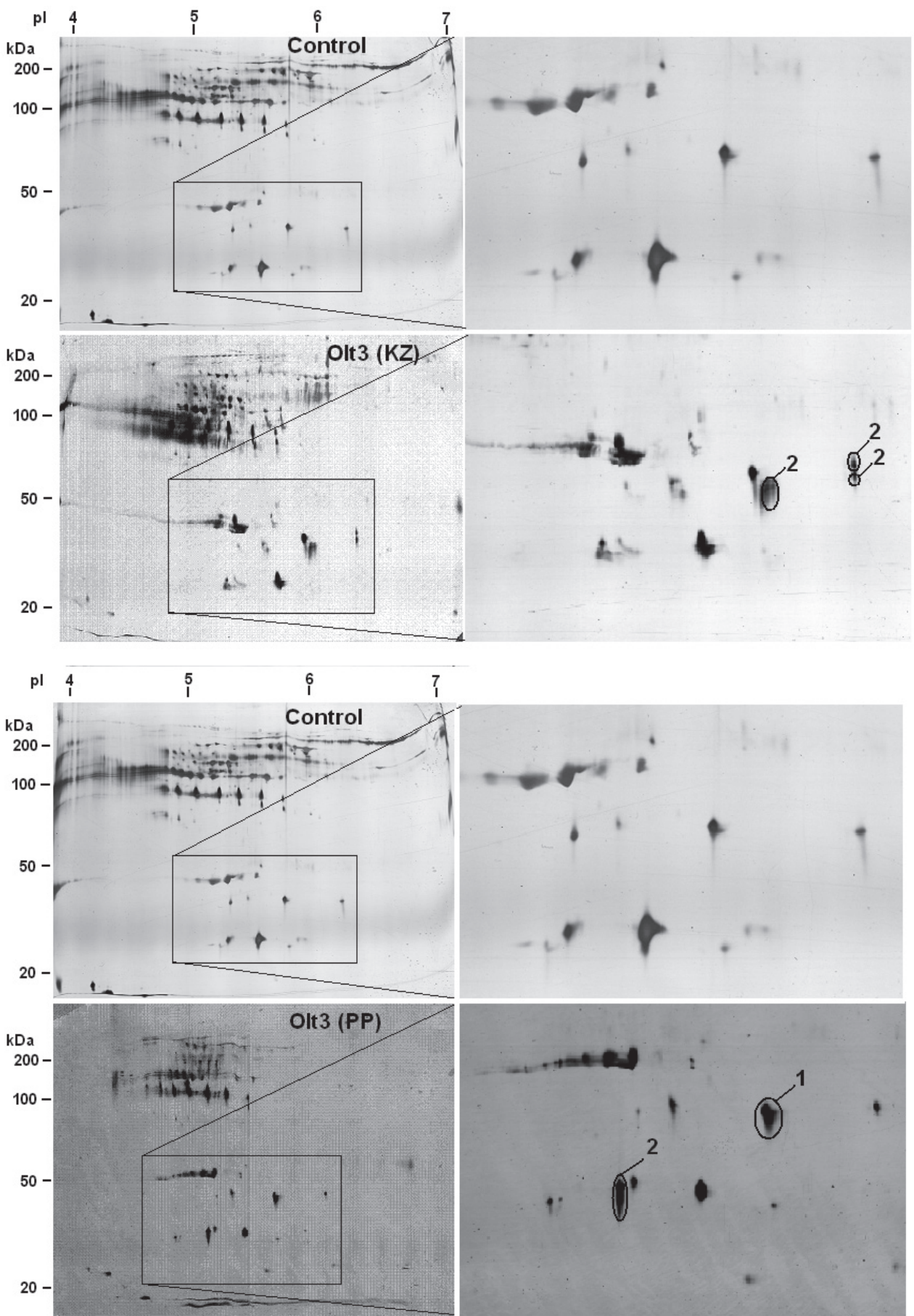

Figure 1A, B. Silver-stained 2D gel electrophoregram of plasma from two children (KZ, PP) with osteogenesis imperfecta type III (Olt3) in comparison with controls.

Panels on the right side show a magnified region of the gels (control, Olt3). The protein spots with significant changes in intensity were labeled with Arabic numbers: 1, human alpha-1 acid glycoprotein; 2, human haptoglobin alpha beta chains.

Quantitative analysis revealed that serum concentration of haptoglobin was significantly higher in children with OIt 3 than in controls; mean values were $4.28 \pm 2.41$ v. $1.17 \pm 1.25 \mathrm{~g} / \mathrm{l}$, respectively, $p=0.003$ (Fig. 3).

\section{DISCUSSION}

Search for the causes of recurrent bone fractures have been going on for a long time and for the time being these causes are still unclear (Manias et al., 2006). At the beginning, decreased bone mineral density was considered the main factor, but after years of observation it appeared not to be the only and sufficient cause of fractures, as fractures frequently occur at normal or only slightly decreased mineral density (Clark et al., 2006). Qualitative disturbances in bone structure, in particular in the collagen matrix, were then suspected. Our study included children with recurrent clinically significant fractures, in whom no disturbances in skeletal mineralization were found with available diagnostic methods, thus raising a suspicion of disturbances in the bone protein 


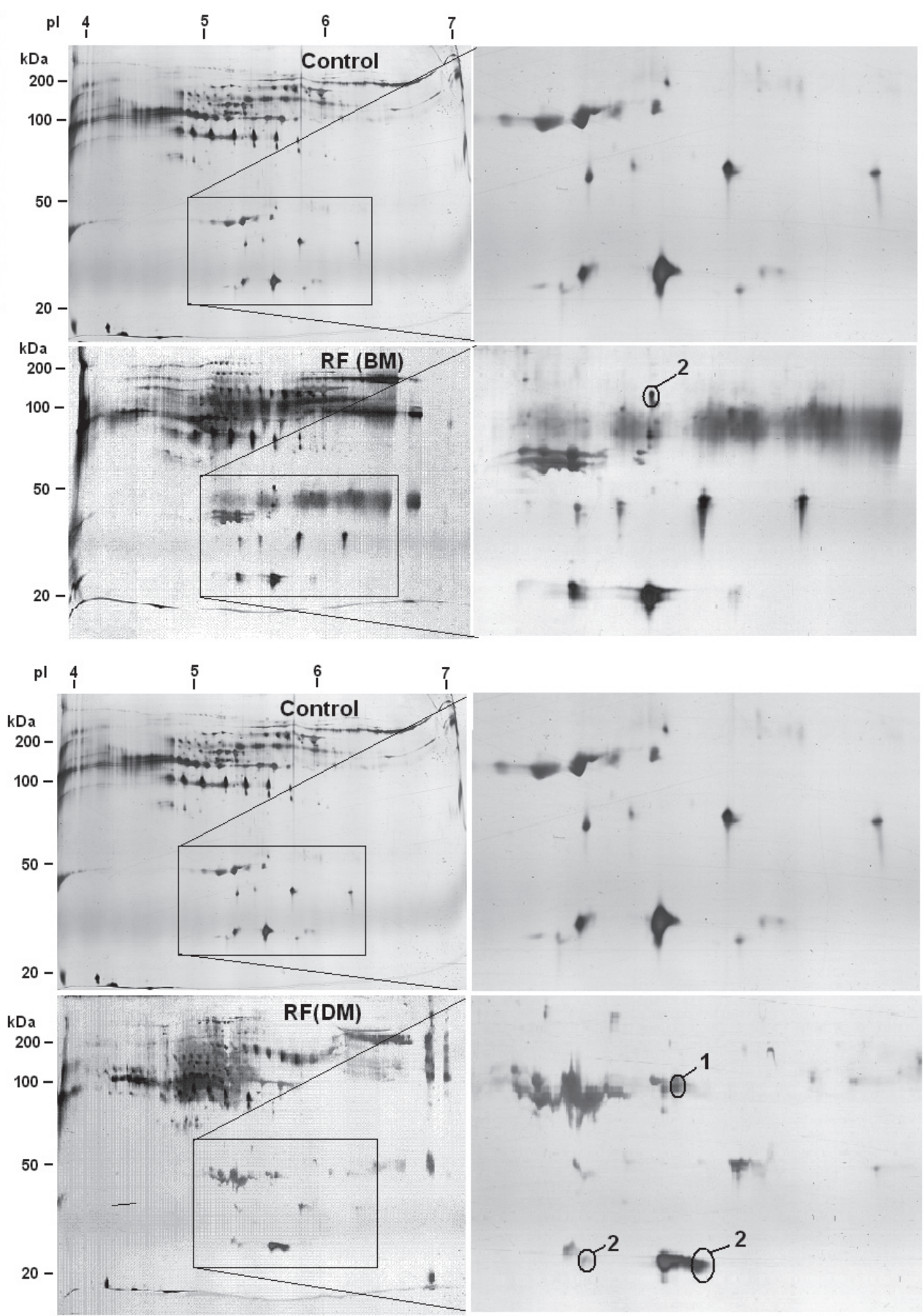

Figure 2A, B. Silver-stained 2D gel electrophoregram of plasma from two children (BM, DM) with recurrent fractures of unknown aetiology (RF) in comparison with controls.

Panels on the right side-show a magnified region of the gels (control, RF). The protein spots with significant changes in intensity were labeled with Arabic numbers: 1, human apolipoprotein A-I; 2, human transthyretin.

component; it also included patients with osteogenesis imperfecta, i.e. with a genetically conditioned disease related to qualitative or quantitative collagen defects.

Analysis of the plasma protein profile with proteomic methods has shown, that haptoglobin is the most frequently occurring protein with changed abundance in children with fractures, as compared to the control group. Our proteomic results were subsequently confirmed by a quantitative ELISA method. Haptoglobin is a glycoprotein built up of alpha and beta chains, with three different phenotypes. Its main function is to bind free haemoglobin and to participate in iron recovery from the haem group; decrease of its concentration is a well-known haemolysis marker (Van Vlierberghe et al., 2004). Most of the iron present in the body comes from haemoglobin metabolism, in which haptoglobin participates (D'Amelio et al., 2008). Previous in vivo studies showed that iron has a significant role also in bone metabolism, including the effect of its abnormal levels on bone demineralisation. In studies done by Campos et al. (1998) on rats fed an iron-deficient diet, disturbances in calcium, phosphate and magnesium metabolism with a significant level of bone demineralisation were observed. Thus it is possible that increased haptoglobin found in 


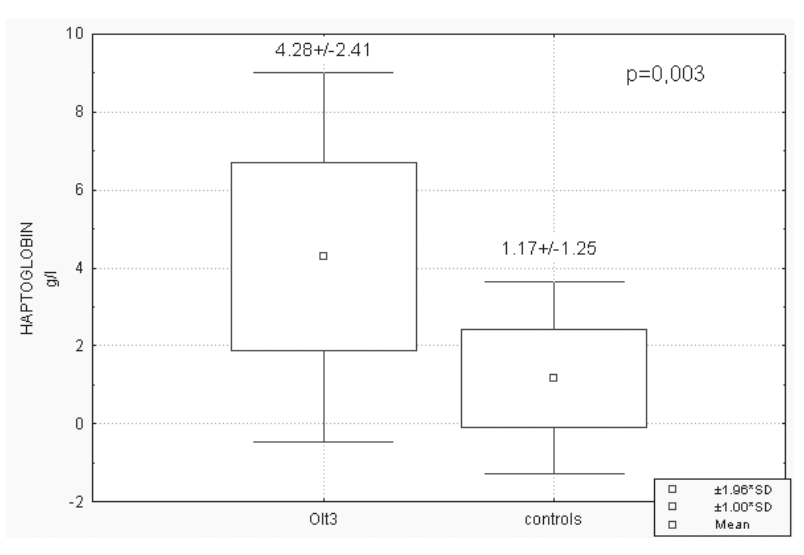

Figure 3. Serum haptoglobin concentration in children with Olt3 and controls $(p<0.05)$.

the studied subjects is associated with disturbances in the iron balance unfavourably affecting bone metabolism. The results obtained in our work also correspond with a recently published report of the team of D'Amelio et al. (2008) who studied the relationship between iron metabolism, including haptoglobin polymorphisms, and postmenopausal osteoporosis. The study revealed that the occurrence of fractures in this group was closely related to haptoglobin phenotype 2.2, i.e. to haptoglobin of the highest molecular weight. These authors also pointed to the fact that haptoglobin 2.2 favours deficiency of iron that is indispensable as a cofactor of enzymes synthesising collagen and bone matrix as well as a cofactor of 25-hydroxylase - an enzyme engaged in activation of vitamin $\mathrm{D}$, and thus in the intestinal calcium absorption. This way they showed a close relationship between haptoglobin, iron metabolism, and the occurrence of fractures in postmenopausal women. Similar relationships may be present in the group of children studied by us and this issue seems to be worth further deepened analysis.

Haptoglobin has also immunomodulatory, antioxidative and angiogenic activity (Delanghe et al., 1999). It is an acute-phase protein, its production in the liver increases in inflammatory conditions and is directly stimulated by proinflammatory proteins, such as interleukin-1, interleukin-6, and tumor necrosis factor alpha (Van Vlierberghe et al., 2004). It is possible that increased expression of this protein in children with particularly numerous fractures, i.e. in children with type III osteogenesis imperfecta, is related with an underlying subclinical inflammatory process. Despite the fact that these children showed no clinical symptoms of inflammation and their C-reactive protein concentration was normal, haptoglobin was the most frequently detected protein with changed expression in their plasma, as compared to the reference group.

Proinflammatory cytokines, in particular interleukin-6, are known to be able to stimulate bone resorption (Carstanjen et al., 2001; Fonseca et al., 2009). This fact is supported by their increased concentration in adults with osteoporosis shown in the literature (Mundy, 2007), as well as by the results of our own previous studies in children with idiopathic osteoporosis (Rusińska \& Chlebna-Sokól, 2005). It is possible that increased expression of haptoglobin in the patients studied by us was accompanied by increased concentration of other proinflammatory factors which enhanced bone resorption and thus increased the risk of fracture. A direct effect of haptoglobin on stimulation of bone resorption is also probable, as this effect was shown in vitro by Lerner and Frohlander (1992).

However, it remains unclear whether increased expression of this acute-phase protein in children with the most numerous fractures was, through increased bone resorption, the cause or rather the effect of the fractures. Nevertheless, to exclude such bias, blood for tests was collected, at the earliest, 2 months after the last fracture, i.e. at the time when it was already healed. Additionally, clinically occult microfractures cannot be excluded that might elicit a discrete inflammatory condition and thus affect obtained results.

As the changed expression of transthyretin, apolipoprotein A-I and serum amyloid P-component was only detected in one or two subjects in the RF and/or the OIt3 group, we will not speculate in this paper about the possible involvement of these proteins in bone fragility, however this issue may need discussion in further studies.

\section{CONCLUSIONS}

The results of pilot studies show increased haptoglobin concentration in children with OIt3, which suggests contribution of an inflammatory component to the course of severe osteogenesis imperfecta.

There is a necessity of further studies to explain the possible relationship of this protein with increased bone fragility.

\section{Acknowledgements}

The study was partly financed by the Ministry of Science and Higher Education as a grant No N N407 060938 and by Medical University of Lodz (No 502-11599 and No 50310 90-2).

\section{Conflict of interest statement}

All authors declare no financial/commercial conflicts of interest.

\section{REFERENCES}

Campos MS, Barrionuevo M, Alferez MJ, Gomez-Ayala AE, Rodriguez-Mata MC, Lopez O (1998) Interactions among iron, calcium, phosphorus and magnesium in the nutritionally iron-deficient rat. Exp Physiol 83: 771-781.

Carstanjen D, Regenfus M, Müller C, Salama A (2001) Interleukin-6 is a major effector molecule of short-term G-CSF treatment inducing bone metabolism and an acute-phase response. Exp Hematol 29: $812-821$.

Clark EM, Tobias JH, Ness AR (2006) Association between bone density and fractures in children: a systematic review and meta-analysis. Pediatrics 117: e291-297.

Cundy T, Horne A, Bolland M, Gamble G, Davidson J (2007) Bone formation markers in adults with mild oseogenesis imperfecta. Clinical Chemistry 6:1109-1114.

D’Amelio P, Cristofaro MA, Tamone C, Morra E, Di Bella S, Isaia G, Grimaldi A, Gennero L, Gariboldi A, Ponzetto A, Pescarmona GP, Isaia GC (2008) Role of iron metabolism and oxidative damage in postmenopausal bone loss. Bone 43: 1010-1015.

D’Ámelio P, Di Bella S, Tamone C, Ravazolli MG, Cristofaro MA, Di Stefano M, Isaia G (2008) HDL cholesterol and bone mineral density in normal-weight postmenopausal women: is there any possible association? Panminerva Med 50: 89-96.

de Ceuninck F (2008) The application of proteomics to articular cartilage: a new hope for the treatment of osteoarthritis. Joint Bone Spine 75: $376-378$. 
Delanghe J, Langlois M, Duprez D, De Buyzere M, Clement D (1999) Haptoglobin polymorphism and peripheral arterial occlusive disease. Atherosclerosis 145: 287-292.

Fonseca JE, Santos MJ, Canhão H, Choy E (2009) Interleukin-6 as a key player in systemic inflammation and joint destruction. Autoimmun Rev 8: 538-542.

Forlino A, Tani C, Rossi A, Lupi A, Campari E, Gualeni B, Bianchi L, Armini A, Cetta G, Bini L, Marini JC (2007) Differential expression of both extracellular and intracellular proteins is involved in the lethal or nonlethal phenotypic variation of BrtlIV, a murine model for osteogenesis imperfecta. Proteomics 7: 1877-1891.

Khosla S, Melton LJ 3rd, Dekutoski MB, Achenbach SJ, Oberg AL, Riggs BL (2003) Incidence of childhood distal forearm fractures over 30 years: a population-based study. JAMA 290: 1479-1485.

Lerner UH, Fröhlander N (1992) Haptoglobin-stimulated bone resorption in neonatal mouse calvarial bones in vitro. Arthritis Rheum 35: 587-591.

Manias K, McCabe D, Bishop N (2006) Fractures and recurrent fractures in children; varying effects of environmental factors as well as bone size and mass. Bone 39: 652-657.

Mayer-Kuckuk P, Boskey AL (2006) Molecular imaging promotes progress in orthopedic research. Bone 39: 965-977.
Mundy GR (2007) Osteoporosis and inflammation. Nutr Rev 65: S147151.

Pernow Y, Hauge EM, Linder K, Dahl E, Sääf M (2009) Bone histomorphometry in male idiopathic osteoporosis. Calcif Tissue Int 84: $430-438$.

Rusińska A, Chlebna-Sokół D (2005) Evaluation of interleukin-1 and -6 in the etiopathogenesis of idiopathic osteoporosis and osteopenia in children. Arch Immunol Ther Exp 53: 257-265.

Shevchenko A, Wilm M, Vorm O, Mann M (1996) Mass spectromeric sequencing of proteins silver-stained polyacrylamide gels. Anal Chem 68: $850-858$.

Van Vlierberghe H, Langlois M, Delanghe J (2004) Haptoglobin polymorphisms and iron homeostasis in health and in disease. Clin Chim Acta 345: 35-42.

Zhang H, Recker R, Lee WN, Xiao GG (2010) Proteomics in bone research. Expert Rev Proteomics 7: 103-111.

Zheng X, Wu SL, Hincapie M, Hancock WS (2009) Study of the human plasma proteome of rheumatoid arthritis. I Chromatogr A 1216: 3538-3545. 